\title{
The Warm Polar Dust in the NLS1 ESO 323-G77
}

\author{
James H. Leftley ${ }^{* a b}$, Konrad R. W. Tristram ${ }^{a}$, Sebastian F. Hönig ${ }^{b}$, Makoto \\ Kishimoto $^{c}$, Daniel Asmus ${ }^{b a}$, and Poshak Gandhi ${ }^{b \dagger}$ \\ ${ }^{a}$ European Southern Observatory, Alonso de Córdova 3107, Casilla 19001, Santiago, Chile \\ ${ }^{b}$ Department of Physics \& Astronomy, University of Southampton, Southampton, SO17 1BJ, UK \\ ${ }^{c}$ Kyoto Sangyo University, Motoyama, Kamigamo, Kita-ku, Kyoto 603-8555, Japan \\ JHL E-mail: jl30g12@soton. ac.uk
}

\begin{abstract}
The dusty torus in AGN unification has long been thought as the source of obscuration in AGN. However, until long baseline IR interferometry, we did not have the capability to directly observe this dusty structure. Advancements in the field of IR interferometry have finally allowed us to get a glimpse of the warm and hot dust structure on the scale of the putative torus. Interferometric studies of the brightest local Seyfert galaxies $(\mathrm{z}<0.05)$ have been undertaken and in those with enough data to discern the angular dependence of the dust distribution, we do not see a simple equatorially-extended structure in the mid-IR. Instead, strong extended dust emission from the polar region is detected in most of the objects, with a subdominant compact or disk-like component in the plane of the accretion disk. In this talk, we will report results of ESO 323-G77, the first Narrow Line Sy1 galaxy (as classified by [1]) to be studied in detail with IR interferometry. While we do find strongly polar-elongated dust emission (axis ratio 3:1) as in other Seyfert galaxies, it is contributing only about $40 \%$ to the total mid-IR emission, and thus not dominant. $60 \%$ of the $12 \mu \mathrm{m}$ flux originates from an unresolved source. Using full IR SED and the 3D radiative transfer model CAT3D-WIND, we interpret that this unresolved mid-IR emission is the Rayleigh-Jeans tail of hot dust emission from the inner part of a dusty disk close to the sublimation radius. These results indicate strong similarities of the NLS1 ESO 323-G77 with the compact emission seen in two quasars [2] and suggest evolution of the dust distribution with Eddington ratio.
\end{abstract}

Revisiting narrow-line Seyfert 1 galaxies and their place in the Universe - NLS1-2018

9-13 April 2018

Padova Botanical Garden, Italy

\footnotetext{
${ }^{*}$ Speaker.

${ }^{\dagger}$ Based on European Southern Observatory (ESO) observing programmes 083.B-0452, 084.B-0366, 087.B0401, 092.B-0718, 095.B-0376, and 290.B-5113.
} 


\section{Introduction}

The distribution of hot, $\approx 1000 \mathrm{~K}$, and warm, $300-400 \mathrm{~K}$, dust in Active Galactic Nuclei (AGN) has come under scrutiny in recent years with improvements in long baseline IR interferometry. Thermal emission from warm dust dominates the mid-IR emission in the central region of AGN peaking in the $8-13 \mu \mathrm{m}$ range. In the classical model of AGN, the bulk of the warm dust is housed in the obscuring dust torus around the equatorial region of the AGN. This torus is responsible for the obscuration required by the unification scheme [3]. With high angular resolution mid-IR interferometers such as the MID-infrared interferometric Instrument, or MIDI [4], and the upcoming Multi-AperTure mid-Infrared SpectroScopic Experiment, or MATISSE [5], we can directly observe AGN on the scale of the dust torus, $1-10 \mathrm{pc}$, for the first time (e.g. [6, 7, 8, 9, 10, 11, 12, 13]). However, instead of a dust torus we see a polar dust extension in both type 1 and type 2 AGN. This polar dust structure cannot be explained with the standard dust torus model. Therefore, a new model, called the disk+wind model, was created which can explain the data. This model comprises of a warm polar dust wind, responsible for the polar extension seen with MIDI, and a hot, geometrically thin, dust disk acting as the dust reservoir [8]. We present the case of ESO 323-G77, the second type 1 and the first NLS1 [1] to be studied in this manner.

\section{Method and Results}

We used unpublished archival data obtained on ESO 323-G77 between 2010 and 2014, together with some previously published material. After reduction and data processing we had 10 uv points which we used to constrain the geometry of ESO 323-G77. By comparing the visibility of each uv point against baseline length we can get an initial idea of the geometry. In Figure 1 we split our observations into 3 groups by PA. The bin sizes and locations were chosen based on data abundance and distribution. The red bin only contains one observation, this is mainly due to technical issues on the baseline for this PA. When we do this we can see a clear drop in visibility with baseline length in each bin. The decrease in all bins appears to level off after 80-100 m, we can interpret this as two separate components. One component is unresolved and therefore constant with baseline and the other is partially resolved. To further constrain the geometry we fit a simple geometric model to the visibility data.

\subsection{Geometric Model}

The visibility was split into wavelength bins of size $0.4 \mu \mathrm{m}$ over the $8 \mu \mathrm{m}$ to $13 \mu \mathrm{m}$ wavelength range, the so-called $\mathrm{N}$ band, offered by MIDI. Each bin was fitted independently. The model consists of an elongated Gaussian and an unresolved source. The unresolved source is a Gaussian with a FWHM of the sublimation radius of ESO 323-G77, 0.321 mas, as predicted by UV measurements [2]. The elongated Gaussian is allowed to fit any PA with equal likelihood and as little geometry was assumed about the source as possible. We utilised an MCMC Bayesian method to fit the model with the PYTHON package EMCEE [14].

The result from the fit of the $11.8 \mu \mathrm{m}$ wavelength bin can be found in Figure 2. The detailed results can be found in Table 1. We find that ESO 323-G77 is highly elongated and has a large, dominant, unresolved source fraction. When we compare the PA of the major axis, on average 


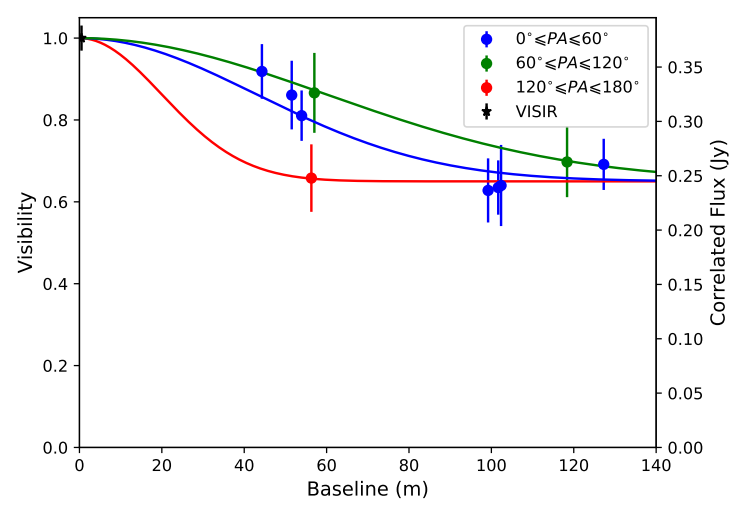

Figure 1: $11.8 \mu \mathrm{m}$ visibility observations binned into three groups solely based on PA and fitted with a Gaussian and a constant. The single red point was given the same constant as the other two fits.

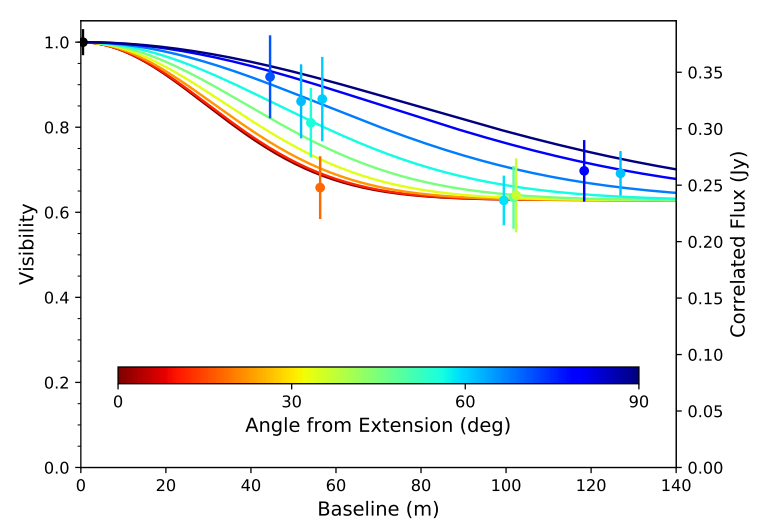

Figure 2: The geometric model fit result of the $11.8 \mu \mathrm{m}$ wavelength bin for ESO 323-G77. The colours depict the angle from the major axis of the extension which has a PA of $155^{\circ}$.

$155^{\circ} \pm 14^{\circ}$, to the polar axis, $174^{\circ} \pm 2^{\circ}$ from polarisation measurements [15], we find that this object is polar extended. This makes ESO 323-G77 the second type 1, and first NLS1, to be studied in this manner and the second to show a strong polar elongation.

\subsection{Radiative Transfer Model}

With more and more interferometric sources being discovered that show polar-elongated midIR emission, a new radiative transfer model of the parsec-scale dusty environment has been developed [16]. The CAT3D-WIND model consists of a dusty disk and a hollow-cone outflow. The parametrisation is a minimal extension to a more generic clumpy torus model and successfully reproduced the mid-IR interferometry and IR SED of NGC 3783, simultaneously.

We followed the same strategy to model the interferometry of ESO 323-G77 as [16]. First, we use the high-resolution IR photometry and spectroscopy of ESO 323-G77 to find acceptable 


\begin{tabular}{|c|c|c|c|c|c|}
\hline Wavelength $(\mu \mathrm{m})$ & $p_{f}$ & $\varepsilon$ & $\Theta_{y}($ mas $)$ & $\theta(\mathrm{deg})$ & $\ln (f)$ \\
\hline 8.2 & $0.79_{0.04}^{0.03}$ & $3.08_{1.24}^{1.27}$ & $30.9_{12}^{46.2}$ & $17170_{47}^{30}$ & $-8.9_{4.1}^{4.3}$ \\
\hline 8.6 & $0.74_{0.04}^{0.03}$ & $3.31_{0.91}^{1.05}$ & $25.9_{5.3}^{10.3}$ & $160_{13}^{19}$ & $-9.8_{3.7}^{4.6}$ \\
\hline 9.0 & $0.76_{0.04}^{0.03}$ & $3.03_{1.11}^{1.29}$ & $26.2_{8.1}^{14.8}$ & $150_{14}^{18}$ & $-9.0_{4.1}^{3.9}$ \\
\hline 9.4 & $0.77_{0.07}^{0.05}$ & $3.01_{1.27}^{1.34}$ & $22.3_{6.9}^{22.4}$ & $182_{27}^{30}$ & $-10.8_{3.1}^{5}$ \\
\hline 9.8 & $0.75_{0.06}^{0.04}$ & $3.09_{1.23}^{1.25}$ & $28.7_{10.6}^{32.4}$ & $155_{33}^{32}$ & $-9.3_{3.9}^{4.3}$ \\
\hline 10.2 & $0.70_{0.04}^{0.03}$ & $2.83_{0.81}^{1.15}$ & $26.6_{5.6}^{7.1}$ & $155_{8}^{13}$ & $-10.5_{3.4}^{4.7}$ \\
\hline 10.6 & $0.67_{0.04}^{0.03}$ & $3.01_{0.82}^{1.112}$ & $26.8_{5.4}^{6.0}$ & $160_{7}^{i 4}$ & $-10.8_{3.2}^{4.6}$ \\
\hline 11.0 & $0.67_{0.04}^{0.03}$ & $2.39_{0.8}^{1.31}$ & $31.1_{7}^{10.6}$ & $152_{12}^{12}$ & $-9.3_{3.9}^{4}$ \\
\hline 11.4 & $0.67_{0.04}^{0.03}$ & $2.63_{0.88}^{1.3}$ & $32.6_{7.7}^{11.3}$ & $154_{11}^{14}$ & $-9.6_{3.7}^{4.2}$ \\
\hline 11.8 & $0.63_{0.04}^{0.03}$ & $2.65_{0.76}^{1.2}$ & $30.9_{6.4}^{8.1}$ & $155_{7}^{16}$ & $-10.6_{3.3}^{4.6}$ \\
\hline 12.2 & $0.63_{0.04}^{0.03}$ & $2.85_{0.91}^{1.26}$ & $36.4_{8.3}^{11.3}$ & $149_{8}^{8}$ & $-9.2_{4}^{4}$ \\
\hline 12.6 & $0.63_{0.03}^{0.03}$ & $2.18_{0.8}^{1.45}$ & $42.0_{12.9}^{26.5}$ & $140_{21}^{22}$ & $-9.6_{3.7}^{4.3}$ \\
\hline 13.0 & $0.62_{0.06}^{0.04}$ & $3.23_{0.99}^{1.09}$ & 28.9 .18 & $170_{13}^{19}$ & $-10.0_{3.6}^{4.6}$ \\
\hline
\end{tabular}

Table 1: $p_{f}$ is the unresolved source fraction, $\varepsilon$ is the ratio of the major axis to minor axis, $\Theta_{y}$ is the major axis FWHM, $\theta$ is the angle from north to east of the $\Theta_{y}$ component of the Gaussian and $f$ is the fractional amount for which the variance is underestimated by the likelihood function if the errors were assumed correct [14].

\begin{tabular}{c|cccccccc}
\hline Parameter & $N_{0}$ & $a_{d}$ & $a_{w}$ & $h$ & $\theta_{w}$ & $\theta_{\sigma}$ & $f_{w d}$ & inc \\
\hline \hline Value & 5 & -3 & -1 & 0.1 & $30^{\circ}$ & $10^{\circ}$ & 0.6 & $60^{\circ}$ \\
\hline
\end{tabular}

Table 2: $N_{0}$ is the average number of clouds in the line of sight in the equatorial region, $a_{d}$ is the radial power law index for the disk $\alpha r^{a_{d}}$ where $\mathrm{r}$ is in units of the sublimation radius, $a_{w}$ is the radial power law index of the dust clouds in the polar wind, $h$ is a unitless disc height scaling factor, $\theta_{w}$ is the opening angle of the polar wind, $\theta_{\sigma}$ is the angular width of the polar wind, $f_{w d}$ is the wind to disk ratio, and inc is the inclination.

fits to the SED from the suite of approximately 132,000 CAT3D-WIND models. Figure 3 shows the observed IR SED in comparison to model SEDs. The light-grey lines in Figure 3 represent all models within the 95\% confidence interval of the best SED fit (solid black line) according to a $\chi^{2}$ distribution. We then compare these models to the interferometric data. This yields only two models that successfully fit both data sets. A satisfactory representation of visibilities has been achieved for the model parameters listed in Table 2. The corresponding model visibilities at $12 \mu \mathrm{m}$ are compared to the data in Figure 4. Within the tested range of parameter steps, those parameters were the only ones to simultaneously reproduce the SED, overall visibility levels, and position angle dependence. The only major degeneracy remained for the width of the hollow cone, $\theta_{\sigma}$, where values of $10^{\circ}$ and $15^{\circ}$ led to similar results.

\section{Conclusion}

Our observations and modelling have revealed highly elongated mid-IR dust emission in ESO 323-G77 with a much higher elongation than those found in previous work [8, 13]. Fur- 


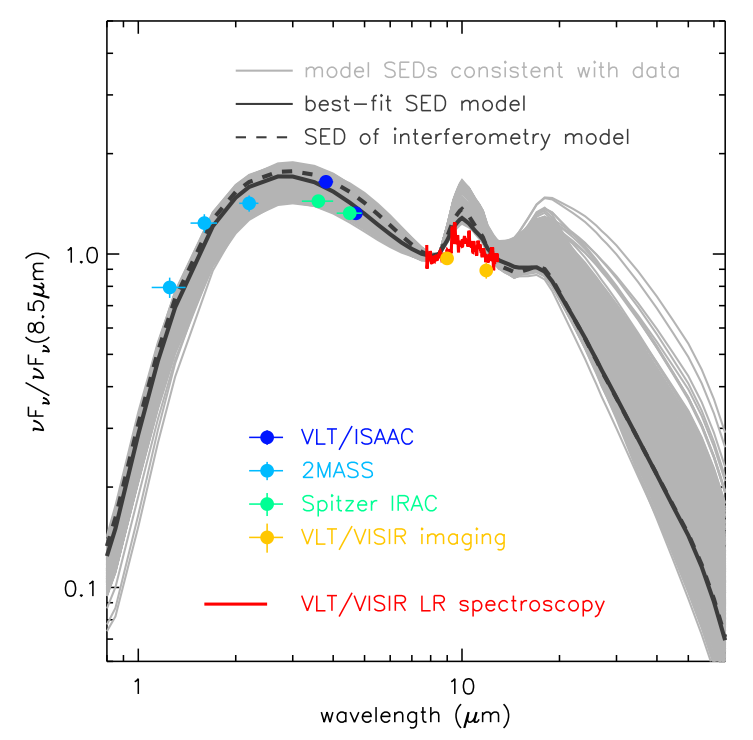

Figure 3: High-resolution SED of ESO 323-G77. The filled circles represent observed photometry from the near-IR and mid-IR while the red line shows the VISIR spectrum. The data have been corrected for Galactic extinction of $A_{V}=1.2$. The light-grey lines show CAT3D-WIND model SEDs that are consistent with the 95\% confidence interval of the best fit (solid black line; see text for details). The dashed black line shows the SED of the model that is used to reproduce the MIDI interferometry in Figure 4.

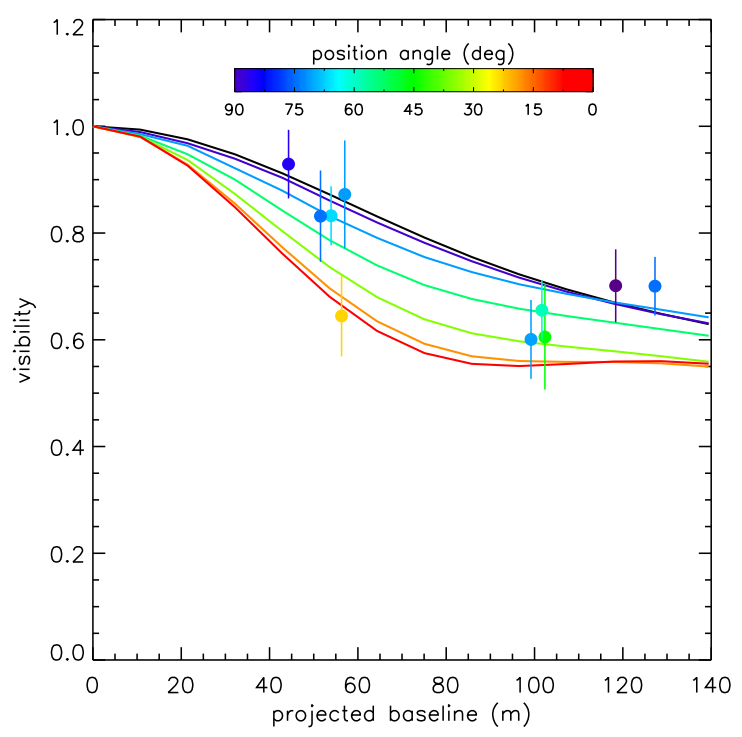

Figure 4: Radial visibility versus baseline as observed (filled circles) and modelled with CAT3D-WIND (solid lines) model at $12 \mu \mathrm{m}$ for ESO 323-G77. The colours represent position angle with respect to $155^{\circ}$, the major axis of the geometric model. 
thermore, ESO 323-G77 is the first clear example of an AGN dominated by unresolved emission in the mid-infrared that, at the same time, has a detected polar extension.

From the full IR SED and the strong unresolved source-wavelength anti-correlation revealed by the geometric modelling, we interpret this dominant unresolved source as the Rayleigh-Jeans tail of the hot dust emission originating from the inner region of the disk close to the sublimation radius. These results indicate strong similarities of ESO 323-G77 with the compact emission seen in two quasars [7]. Given that ESO 323-G77 is considered having a higher Eddington ratio than typical Seyferts (relatively narrow Hydrogen emission lines in the optical), similar to quasars, our result hints at evolution of the geometric dust distribution around an AGN with Eddington ratio. Indeed, such a scenario is consistent with radiation pressure shaping the mass distribution on the sub-parsec to parsec-scale environment of the AGN [17, 18].

\section{Acknowledgements}

This conference has been organized with the support of the Department of Physics and Astronomy "Galileo Galilei", the University of Padova, the National Institute of Astrophysics INAF, the Padova Planetarium, and the RadioNet consortium. RadioNet has received funding from the European Union's Horizon 2020 research and innovation programme under grant agreement No 730562. JHL, SFH, and DA acknowledge support from the Horizon 2020 ERC Starting Grant DUST-IN-THE-WIND (ERC-2015-StG-677117). PG thanks STFC for support (grant reference ST/J003697/2). MK acknowledges support from JSPS under grant 16H05731.

\section{References}

[1] E. Sani, D. Lutz, G. Risaliti, H. Netzer, L. C. Gallo, B. Trakhtenbrot et al., Enhanced star formation in narrow-line Seyfert 1 active galactic nuclei revealed by Spitzer, Monthly Notices of the Royal Astronomical Society 403 (2010) 1246.

[2] M. Kishimoto, S. F. Hönig, R. Antonucci, R. Barvainis, T. Kotani, K. R. W. Tristram et al., The innermost dusty structure in active galactic nuclei as probed by the Keck interferometer, Astronomy and Astrophysics 527 (2011) A121.

[3] R. Antonucci, Unified models for active galactic nuclei and quasars., Annual Review of Astronomy and Astrophysics 31 (1993) 473.

[4] C. Leinert, U. Graser, F. Przygodda, L. B. F. M. Waters, G. Perrin, W. Jaffe et al., MIDI - the 10 $\$ \backslash m u \$ m$ instrument on the VLTI, Astrophysics and Space Science 286 (2003) 73.

[5] B. Lopez, P. Antonelli, S. Wolf, S. Lagarde, W. Jaffe, R. Navarro et al., MATISSE: perspective of imaging in the mid-infrared at the VLTI, in Optical and Infrared Interferometry, vol. 7013, p. 70132B, International Society for Optics and Photonics, July, 2008, DOI.

[6] K. R. W. Tristram, Mid-infrared interferometry of nearby Active Galactic Nuclei, 2007.

[7] M. Kishimoto, S. F. Hönig, R. Antonucci, F. Millour, K. R. W. Tristram and G. Weigelt, Mapping the radial structure of AGN tori, Astronomy $\backslash \&$ Astrophysics 536 (2011) A78.

[8] S. F. Hönig, M. Kishimoto, R. Antonucci, A. Marconi, M. A. Prieto, K. Tristram et al., Parsec-scale Dust Emission from the Polar Region in the Type 2 Nucleus of NGC 424, The Astrophysical Journal 755 (2012) 149. 
[9] S. F. Hönig, M. Kishimoto, K. R. Tristram, M. A. Prieto, P. Gandhi, D. Asmus et al., Dust in the polar region as a major contributor to the infrared emission of active galactic nuclei, The Astrophysical Journal 771 (2013) 87.

[10] L. Burtscher, K. Meisenheimer, K. R. W. Tristram, W. Jaffe, S. F. Hönig, R. I. Davies et al., A diversity of dusty AGN tori - Data release for the VLTI/MIDI AGN Large Program and first results for 23 galaxies, Astronomy \& Astrophysics 558 (2013) A149.

[11] K. R. W. Tristram, L. Burtscher, W. Jaffe, K. Meisenheimer, S. F. Hoenig, M. Kishimoto et al., The dusty torus in the Circinus galaxy: a dense disk and the torus funnel, Astronomy and Astrophysics $\mathbf{5 6 3}$ (2014) .

[12] S. F. Hönig, D. Watson, M. Kishimoto and J. Hjorth, A dust-parallax distance of 19 megaparsecs to the supermassive black hole in NGC 4151, Nature 515 (2014) 528.

[13] N. López-Gonzaga, L. Burtscher, K. R. W. Tristram, K. Meisenheimer and M. Schartmann, Mid-infrared interferometry of 23 AGN tori: On the significance of polar-elongated emission, Astronomy \& Astrophysics 591 (2016) A47.

[14] D. Foreman-Mackey, D. W. Hogg, D. Lang and J. Goodman, emcee: The MCMC Hammer, Publications of the Astronomical Society of the Pacific 125 (2013) 306.

[15] H. M. Schmid, I. Appenzeller and U. Burch, Spectropolarimetry of the borderline Seyfert 1 galaxy ESO 323-G077, Astronomy and Astrophysics 404 (2003) 505.

[16] S. F. Hönig and M. Kishimoto, Dusty Winds in Active Galactic Nuclei: Reconciling Observations with Models, The Astrophysical Journal Letters 838 (2017) L20.

[17] A. C. Fabian, R. V. Vasudevan and P. Gandhi, The effect of radiation pressure on dusty absorbing gas around active galactic nuclei, Monthly Notices of the Royal Astronomical Society 385 (2008) L43.

[18] C. Ricci, B. Trakhtenbrot, M. J. Koss, Y. Ueda, K. Schawinski, K. Oh et al., The close environments of accreting massive black holes are shaped by radiative feedback, Nature 549 (2017) 488. 